\title{
Using natural variation to investigate the function of individual amino acids in the sucrose-binding box of fructan:fructan 6G-fructosyltransferase (6G-FFT) in product formation
}

\author{
Tita Ritsema ${ }^{1,2, *}$, Auke Verhaar ${ }^{1}$, Irma Vijn ${ }^{1}$ and Sjef Smeekens ${ }^{1}$ \\ ${ }^{1}$ Department of Molecular Plant Physiology, University Utrecht, Padualaan 8, 3584, Utrecht, CH, The \\ Netherlands (*author for correspondence; e-mail Tita.Ritsema@unibas.ch); ${ }^{2}$ Present address: Zürich Basel \\ Plant Science Center, Botanisches Institut der Universität Basel, Hebelstrasse 1, 4056, Basel, Switzerland
}

Received 27 January 2005; accepted in revised form 26 April 2005

Key words: fructan, inulin neo-series, natural variation, site-directed mutagenesis, sucrose-binding box

\begin{abstract}
Enzymes of the glycosyl hydrolase family 32 are highly similar with respect to primary sequence but catalyze divergent reactions. Previously, the importance of the conserved sucrose-binding box in determining product specificity of onion fructan:fructan $6 \mathrm{G}$-fructosyltransferase (6G-FFT) was established [Ritsema et al., 2004, Plant Mol. Biol. 54: 853-863]. Onion 6G-FFT synthesizes the complex fructan neoseries inulin by transferring fructose residues to either a terminal fructose or a terminal glucose residue. In the present study we have elucidated the molecular determinants of product specificity by substitution of individual amino acids of the sucrose binding box with amino acids that are present on homologous positions in other fructosyltransferases or vacuolar invertases. Substituting the presumed nucleophile Asp85 of the $\beta$-fructosidase motif resulted in an inactive enzyme. 6G-FFT mutants S87N and S87D did not change substrate or product specificities, whereas mutants N84Y and N84G resulted in an inactive enzyme. Most interestingly, mutants N84S, N84A, and N84Q added fructose residues preferably to a terminal fructose and hardly to the terminal glucose. This resulted in the preferential production of inulin-type fructans. Combining mutations showed that amino acid 84 determines product specificity of $6 \mathrm{G}-\mathrm{FFT}$ irrespective of the amino acid at position 87 .
\end{abstract}

Abbreviations: 1-SST, sucrose:sucrose 1-fructosyltransferase; 1-FFT, fructan:fructan 1-fructosyltransferase; 6-SFT, sucrose:fructan 6-fructosyltransferase; 6G-FFT, fructan:fructan 6G-fructosyltransferase; aa, amino acid; DP, degree of polymerization; GH32, glycosyl hydrolase family 32

\section{Introduction}

Fructans are part of the human diet and are naturally present in a number of plants consumed by humans, including onion, leek, and artichoke. Studies on the health aspects of a high fructancontaining diet have resulted in the acceptance of fructans as a health-promoting food ingredient (Roberfroid, 2002).
Fructosyltransferases synthesize fructan from sucrose. In plants at least two enzymes are needed to synthesize fructans, one synthesizes the shortest fructan 1-kestose from sucrose, the other elongate 1-kestose (Edelman and Jefford, 1968; Koops and Jonker, 1996; Lüscher et al., 1996; Van den Ende and Van Laere, 1996). The 1-kestose synthesizing enzyme is called sucrose:sucrose 1-fructosyltransferase (1-SST, EC 2.4.1.99). It attaches a fructose 
to the fructose residue of sucrose via a $\beta(2-1)$ linkage and is present in all fructan synthesizing plants. In dicots, fructan:fructan 1-fructosyltransferase (1-FFT, EC 2.4.1.100) repeatedly elongates 1-kestose on its terminal fructose with a $\beta(2-1)$ linked fructose residue producing inulin-type fructans. In grasses sucrose:fructan 6-fructosyltransferase (6-SFT, EC 2.4.1.10) uses 1-kestose to synthesize bifurcose (1\&6-kestotetraose), the shortest branched fructan (Sprenger et al., 1995). In onion, fructan:fructan 6G-fructosyltransferase (6G-FFT) utilizes 1-kestose to synthesize fructans of the neo-series inulin (Vijn et al., 1997). Expression of onion 6G-FFT in tobacco BY2 cells followed by analysis of its activity showed that 6G-FFT has dual transfer specificities coupling fructosyl residues to either the terminal glucose via a $\beta(2-6)$ linkage or a terminal fructose via a $\beta(2-1)$ linkage (Ritsema et al., 2003). Recently these observations were confirmed with 6G-FFT purified from onion (Fujishima et al., 2005). As a result of its two activities 6G-FFT generates several types of fructan chains (1) an inulin series which is elongated at the fructose of the starter sucrose, designated Ix (x represents the degree of polymerization); (2) a neokestose based series with elongation only at the glucose residue of the starter sucrose, designated $N x$; (3) another neokestose based series with elongation on both the fructose and glucose residue of the starter sucrose, designated Nx (Ernst et al., 1998) (Figure 1). Furthermore, the fructan profile observed in onion extracts resembles that of 1-kestose fed 6G-FFT, providing evidence for the hypothesis that besides 1-SST 6G-FFT is the only fructosyltransferase present in onion.
Fructosyltransferases probably evolved from vacuolar invertases ( $\beta$-fructofuranosidase, EC 3.2.1.26) (Hendry, 1993; Wei et al., 2001). Invertases are present in all plants where they hydrolyze sucrose into glucose and fructose. Invertases and fructosyltransferases share the so-called sucrose-binding box, a motif that was first observed in invertases and that is essential for sucrose binding (Reddy and Maley, 1990). Vacuolar invertases and fructosyltransferases are homologous in their primary structure with several invariant boxes. Interestingly, they differ in their sucrose-binding boxes. The $\beta$-fructosidase motif encoded within the sucrose-binding box of invertases has the consensus NDPNG/A. The aspartic acid (D) of this motif is the presumed nucleophile involved in substrate binding. In fructosyltransferases alterations of the $\beta$-fructosidase motif are present at both asparagine $(\mathrm{N})$ residues. A variety of amino acids is present in these positions, for example, serine (S), aspartic acid (D), tyrosine $(\mathrm{Y})$, and glycine $(\mathrm{G})$ (Tables 1 and 2). Compared to invertases, the second asparagine in onion 6G-FFT is changed into serine. Sucrose-binding boxes of fructosyltransferases show a much larger sequence variation than sucrose-binding boxes of invertases. This could be linked to the larger enzymatic variability seen for fructosyltransferases. Interesting in this respect is that similarities can be observed between enzymes catalyzing the same reactions. It suggests a link between the sucrose-binding box sequence and the type of substrate that is accepted or product that is made. In addition, there is also a divergence in enzymes from monocot and dicot plants. The $\beta$-fructosidase motifs of the sucrose-binding

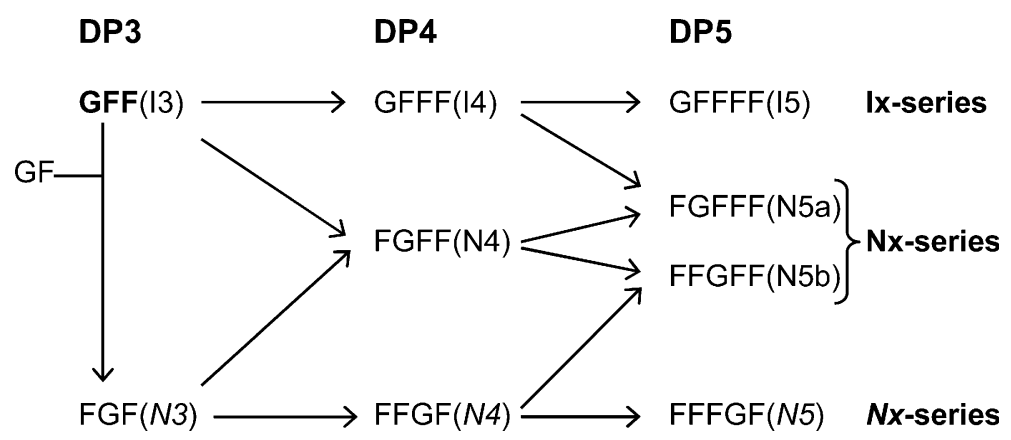

Figure 1. Scheme of the synthesis of different onion-type fructans by 6G-FFT. Every step uses 1-kestose or a higher DP fructan, as the fructosyl donor. I3, 1-kestose; N3, 6G-kestotriose; I4, 1,1-kestotetraose; N4, 1,6G-kestotetraose; N4, 1\&6G-kestotetraose; I5, 1,1,1-kestopentaose; N5, 1,1,6G-kestopentaose; N5a, 1,1\&6G-kestopentaose; N5b, 1\&1,6G-kestopentaose. 
Table 1. Sucrose-binding box sequences of various vacuolar invertases and fructosyltransferases.

\begin{tabular}{|c|c|c|c|}
\hline Plant species (mono/dicot) & Enzyme & Sucrose-binding box & (aa nr.) \\
\hline Arabidopsis thaliana $(\mathrm{d})$ & vac.INV & HFQPEQNWMNDPNG & 134 \\
\hline Beta vulgaris $(\mathrm{d})$ & vac.INV & HFQPQKNWMNDPNG & 160 \\
\hline Cichorium intybus (d) & vac.INV & HFQPKKNWMNDPNG & 141 \\
\hline Daucus carota $(\mathrm{d})$ & vac.INV & HFQPQENWMNDPNG & 148 \\
\hline Ipomoea batatas (d) & vac.INV & HFQPEKNWMNDPNG & 148 \\
\hline Nicotiana tabacum (d) & vac.INV & HFQPQKNWMNDPNG & 129 \\
\hline Lycopersicon pimpinellifolium (d) & vac.INV & HFQPQKNWMNDPNG & 121 \\
\hline Allium сера $(\mathrm{m})$ & vac.INV & HFQPVKNWMNDPNG & 167 \\
\hline Asparagus officinale (m) & vac.INV & HFQPEKNWMNDPNG & 135 \\
\hline Lolium temulentum (m) & vac.INV & HFQPEKNWMNDPNG & 151 \\
\hline Oryza sativa $(\mathrm{m})$ & vac.INV & HFQPEHNWMNDPNG & 142 \\
\hline Zea mays $(\mathrm{m})$ & vac.INV & HFQPPKNWMNDPNG & 135 \\
\hline Allium cepa $(\mathrm{m})$ & 6G-FFT & HFRTVRNYMNDPSG & 88 \\
\hline Cichorium intybus (d) & 1-FFT & HFQPAKNFIYDPNG & 107 \\
\hline Cynara scolymnus (d) & 1-FFT & HFQPAKNFIYDPNG & 107 \\
\hline Helianthus tuberosus (d) & 1-FFT & HFQPAKNFIYYPDG & 105 \\
\hline Agropyron cristatum (m) & 6-SFT & HFQPAKNYMSDPNG & 104 \\
\hline Hordeum vulgare $(\mathrm{m})$ & 6-SFT & HFQTAKNYMSDPNG & 99 \\
\hline Lolium perenne (m) & 6-SFT & HYQPEGHFMSDPNG & 107 \\
\hline Poa secunda $(\mathrm{m})$ & 6-SFT & HFQTEKNFMSDPNG & 99 \\
\hline Triticum aestivum $(\mathrm{m})$ & 6-SFT & HFQTAKNYMSDPNG & 97 \\
\hline Cichorium intybus (d) & 1-SST & HFQPDKNFISDPDG & 123 \\
\hline Cynara scolymnus (d) & 1-SST & HFQPDKNYISDPDG & 120 \\
\hline Helianthus tuberosus (d) & 1-SST & HFQPDKNFISDPDG & 111 \\
\hline Taraxacum officinale (d) & 1-SST & HFQPDKNFISDPDG & 115 \\
\hline Allium сера $(\mathrm{m})$ & 1-SST & HFQPPNHFMADPNA & 100 \\
\hline Allium sativum $(\mathrm{m})$ & 1-SST & HFQPPYHFMGDPNA & 100 \\
\hline Festuca arundinaceae (m) & 1-SST & HFQPEKHYMNDPNG & 139 \\
\hline Lolium perenne $(\mathrm{m})$ & 1-SST & HFQPLKHYMNDPNG & 129 \\
\hline Triticum aestivum (m) & 1-SST & HFQPDKYYQNDPNG & 139 \\
\hline
\end{tabular}

The $\beta$-fructosidase motif is written in bold. $(\mathrm{m})$ and $(\mathrm{d})$ indicates whether plants are monocots $(\mathrm{m})$ or dicots (d). Accession numbers; A. thaliana INV, S71276; B. vulgaris INV, AJ422051; C. intybus INV, AJ419971; D. carota INV, X75352; I. batatas INV, AAD01606; N. tabacum INV, AJ305044; L. pimpinellifolium INV, Z12026; A. cepa INV, AJ006067; A. officinale INV, AF002656; L. temulentum INV, CAD58681; O. sativa INV, AY037870 ; Z. mays INV, U16123; A. cepa 6G-FFT, ACY07838; C. intybus 1-FFT, U84398; C. scolymnus 1-FFT, AJ000481; H. tuberosus 1-FFT, AJ009756; A. cristatum 6-SFT, AF211253; H. vulgare 6-SFT, X83233; L. perenne 6-SFT, AF494041; P. secunda 6-SFT, AF192394; T. aestivum 6-SFT, AB029887; C. intybus 1-SST, U81520; C. scolymnus 1-SST, Y09662; H. tuberosus 1-SST, AJ009757; T. officinale 1-SST, AJ250634; A. cepa 1-SST, AJ006066; A. sativum 1-SST, AY098442; F. arundinaceae 1-SST, AJ297369; L. perenne 1-SST, AF492836; T. aestivum 1-SST, AB029888.

boxes as such can already be grouped according to enzyme and origin. Dicot 1-SSTs contain SDPDG as their $\beta$-fructosidase motif, 6-SFTs contain SDPNG, and 1-FFTs have YDP[ND]G
(Table 1). Whether these differences in motifs imply different fructosyl transfer reactions is an important question that led to the research described in this paper.

Table 2. Sequence variation in sucrose-binding boxes. Compared are the sucrose-binding box sequences of glycosyl hydrolase family 32 as presented by Pons et al. (2000), plant vacuolar invertases, and fructosyltransferases.

\begin{tabular}{lllllllllllllll}
\hline aa nr. in box & 1 & 2 & 3 & 4 & 5 & 6 & 7 & 8 & 9 & 10 & 11 & 12 & 13 & 14 \\
\hline GH32 & H & & & PTV & & & & LIVMA & NSCAYG & DE & P & NDSC & GA \\
vacuolar invertase & H & & Q & PT & & HN & W & M & N & D & P & N & G \\
fructosyl transferase & H & FY & QR & PT & & & HNY & FY & IMQ & NSAYG & D & P & NDS & GA \\
6G-FFT & H & F & R & T & V & R & N & Y & M & N & D & P & S & G \\
\hline
\end{tabular}

The amino acids in the 6G-FFT sucrose-binding box are provided. 
Previously, we showed that substituting the $\beta$-fructosidase motif of the sucrose-binding box of onion 6G-FFT for that of onion 1-SST resulted in a change in products synthesized from the substrate 1-kestose. Changing the $\beta$-fructosidase motif of 6G-FFT in that of invertase did not result in any change (Ritsema et al., 2004). Here we analyze the function of individual amino acids in the sucrose-binding box to obtain insight into the underlying principles of enzymatic specificity. Interestingly, we observed that amino acid 84 determines product specificity of 6G-FFT.

\section{Materials and methods}

Site-directed mutagenesis of 6G-FFT

Mutants were made according to the Altered sites II mutagenesis kit (Promega). The first 1337 bases of the 6G-FFT gene were cloned from pBlue into pALTER-1 using EcoRI. The mutagenesis was confirmed by sequencing. For expression in plants, the mutated gene was cloned into a pMON999 that already contained 3' 6G-FFT (from the KpnI site) using $E c o$ RI, and the orientation was checked by restriction analysis. To enable Agrobacterium-mediated transformation the cassette containing the gene with $35 \mathrm{~S}$ promoter and NOS terminator sequence was excised from pMON999 using NotI, and ligated to Bsp120I digested pAPV1 (Ritsema et al., 2003).

\section{Growth and transformation of BY2 cells}

BY2 cells were grown as a suspension culture in modified Linsmaier and Skoog medium as described by Nagata et al. (1992). The cells were grown at $27^{\circ} \mathrm{C}$ at $150 \mathrm{rpm}$ and sub cultured once a week by a 70 -fold dilution in fresh medium.

Agrobacterium-mediated transformation of BY2 cells was performed according to An (1985) and Genschik et al. (1998) as described before (Ritsema et al., 2003). Transformed callus was maintained by transferring small calli pieces to a fresh plate with kanamycin $(100 \mu \mathrm{g} / \mathrm{ml})$ and vancomycin $(750 \mu \mathrm{g} / \mathrm{ml})$ once a month.

\section{Assay for fructosyltransferase activity}

Fructosyltransferases were isolated from growing BY2 callus by shaking Eppendorf tubes containing cells in a dismembrator (B. Braun Biotech International) two times for $1 \mathrm{~min}$ at $2800 \mathrm{rpm}$ in the presence of glass beads ( 2 of $\varnothing 2 \mathrm{~mm}$ and 2 of $\varnothing$ $4 \mathrm{~mm}$ ). Debris was spun down at $13000 \mathrm{rpm}$ for $15 \mathrm{~min} .40 \mu \mathrm{l}$ of the supernatant was combined with $5 \mu \mathrm{l}$ of an $0.5 \mathrm{M}$ MES buffer pH5,7 and $5 \mu \mathrm{l}$ of substrate to a total volume of $50 \mu \mathrm{l}$, and incubated at $28^{\circ} \mathrm{C}$. After incubation, products were analysed using a Dionex HPAEC-PAD (high performance anion exchange chromatography with pulsed amperometric detection) system with a PA100 column (Ritsema et al., 2003). Solutions were: (A) water; (B) $0.5 \mathrm{M} \mathrm{NaOH}$; and (C) $1 \mathrm{M}$ NaAc. Running profile applied: $T=0,80 \%$ A $20 \% \mathrm{~B} ; T=5 \mathrm{~min}, 50 \%$ A $50 \% \mathrm{~B} ; T=15 \mathrm{~min}$, $40 \%$ A $50 \%$ B $10 \%$ C; $T=20 \mathrm{~min}, 33 \%$ A $50 \%$ B $17 \% \mathrm{C} ; T=35 \mathrm{~min}, 50 \%$ B $50 \% \mathrm{C}$. We used the peak identification as proposed by Shiomi (1993) and Shiomi et al. (1997) and Ernst et al. (1998) and nomenclature as proposed by Waterhouse and Chatterton (1993). All experiments were at least preformed two times; a representative sample is shown in the figures.

\section{Results}

Sucrose-binding box consensus in vacuolar invertases and fructosyltransferases

Plant fructosyltransferases belong to family 32 of the glycosyl hydrolases. The sucrose-binding box consensus of GH32 was determined to consist of H-X-X-[PTV]-X-X-X-X-[LIVMA]-[NSCAYG][DE]-P-[NDSC]-[GA] (Table 2) (Pons et al., 2000).

When the subgroup of plant vacuolar invertases are compared (Table 1), less variation is seen. The variability observed in the sucrose-binding box of vacuolar invertases is limited to H-F-Q[PT]-X-X-[HN]-W-M-N-D-P-N-G. It is striking that $\beta$-fructosidase motif is consistently NDPNG and that the highly variable second, third, and eighth amino acids of the general consensus are invariable in vacuolar invertases being Phe (F), Gln (Q) and Trp (W) respectively.

In fructosyltransferases a wider variety of amino acids is seen than for vacuolar invertases giving the motif $\mathrm{H}-[\mathrm{FY}]-[\mathrm{QR}]-[\mathrm{PT}]-\mathrm{X}-\mathrm{X}-[\mathrm{HNY}]-$ [FY]-[IMQ]-[NSAYG]-D-P-[NDS]-[GA] (Table 2). Compared to vacuolar invertases the $\beta$-fructosidase motif, as well as the second, third, and eighth 
amino acid is not invariable any more. A striking difference between invertases and fructosyltransferases lies in the aromatic eighth amino acid. In invertases a $\operatorname{Trp}(\mathrm{W})$ is invariably present at this position, however in fructosyltransferases there is never a Trp, although there is always a aromatic amino acid in this position, which can be either Tyr (Y) or Phe (F).

The invariable Asp (D) is the proposed nucleophile of invertases involved in sucrose binding (Reddy and Maley, 1996; Alberto et al., 2004).

\section{Asn84 in the $\beta$-fructosidase motif of 6G-FFT determines product preference}

In onion 6G-FFT the sucrose-binding box consists of HFRTVRNYMNDPSG in which the $\beta$-fructosidase motif is NDPSG. This $\beta$-fructosidase motif differs from the general consensus for invertases, NDPN[GA]. In a previous paper (Ritsema et al., 2004) we reported the 6G-FFT mutant S87N, with the $\beta$-fructosidase motif NDPNG, to have the same enzymatic specificity as wild-type 6G-FFT (NDPSG).

The observed natural variation in $\beta$-fructosidase motifs was used as a guide to further design mutants in 6G-FFT. The 6G-FFT mutant S87D was made to resemble the amino acid present in dicot 1-SSTs (Table 1). In addition, the double mutant N84S;S87D was made to obtain SDPDG, the $\beta$-fructosidase motif of dicot 1-SSTs. Incubation of S87D with 1-kestose led to the synthesis of both inulin (Ix) and neo-series inulin (Nx and $N x$ ) products as seen for wild-type 6G-FFT (Figures 2B and 3, Table 3). In contrast, the double mutant N84S;S87D showed an altered product profile. It has a preference for the synthesis of inulin-type fructans (Ix series) (Figures 1, 2B, 3, Table 3). Incubation of the enzymes with sucrose did not lead to polymerized products.

To investigate whether the altered product profile was the result of the combination of mutations, or of the N84S mutation on its own, we introduced N84S in 6G-FFT, resulting in the $\beta$ fructosidase motif SDPSG. N84S showed a preference for Ix series fructan synthesis and thus behaves similar as N84S;S87D (Figure 2B, C, Table 3). This indicates that the altered profile is due to the N84S mutation and that the S87D mutation does not affect product specificity. However, the N84S mutant appears to be able to produce longer fructans than the N84S;S87D mutant (Figure 3). This is especially observed after 4 days of incubation with 1-kestose where N84S made I6 and higher DP fructans at the cost of I4, whereas N84S;S87D made hardly any I6 and has as a consequence more I4 left.

In addition to the above mentioned combinations also 6G-FFT N84S;S87N, resulting in the 6SFT-like motif SDPNG, was tested (Figure 2C). This mutant has the same profile as N84S and N84S;S87D (Table 3) since the main fructan series produced is the Ix series. Like N84S it is able to produce relatively long fructans (Figure 3 ).

The profile of N84S;S87D is highly similar to that of the previously reported mutant N84A; $\mathrm{S} 87 \mathrm{~N}$, which has the $\beta$-fructosidase motif ADPNG similar to that of onion 1-SST (Figures 2D and 3, Table 3) (Ritsema et al., 2004). According to the results described above, mutation $\mathrm{S} 87 \mathrm{~N}$ might not influence product preference of 6G-FFT wild-type and mutants. Therefore we investigated whether the N84A mutation is responsible for the productprofile observed with 6G-FFTN84A;S87N. For this the 6G-FFT single mutant N84A was constructed, and this mutant indeed shows the same product profile as the $6 \mathrm{G}-\mathrm{FFTN} 84 \mathrm{~A} ; \mathrm{S} 87 \mathrm{~N}$ double mutant (Figures 2D and 3, Table 3). This implies that N84A is responsible for a shift in product profile towards Ix-series fructans. In this respect the 6G-FFT N84A and N84S mutants result in the same shift in specificity.

Other mutations of 6G-FFT N84 were tested as well. Based on the $\beta$-fructosidase motif of garlic 1-SST the mutant 6G-FFT N84G was made; and based on dicot 1-FFTs mutant 6G-FFT N84Y was made (Table 1). However, these two mutants showed no activity (Table 3). The 6G-FFT N84Y;S87N double mutant, which has YDPNG as the $\beta$-fructosidase motif resembling that of 1 -FFTs, is also inactive (Table 3 ).

The closest structurally homologous amino acid of Asn is Gln, therefore 6G-FFT N84Q was made. 6G-FFT N84Q showed a strong preference for synthesis of 1,1-kestotetraose (I4) from 1-kestose (Figures 2E and 3, Table 3). Interestingly, it showed the most limited product profile of all mutations tested, since prolonged incubation did not result in elongation of 1,1-kestotetraose. Additionally, the ability of N84Q to elongate 1,1kestotertraose was tested using I4 as a substrate in concentrations up to $200 \mathrm{mM}$ and for assays up to 

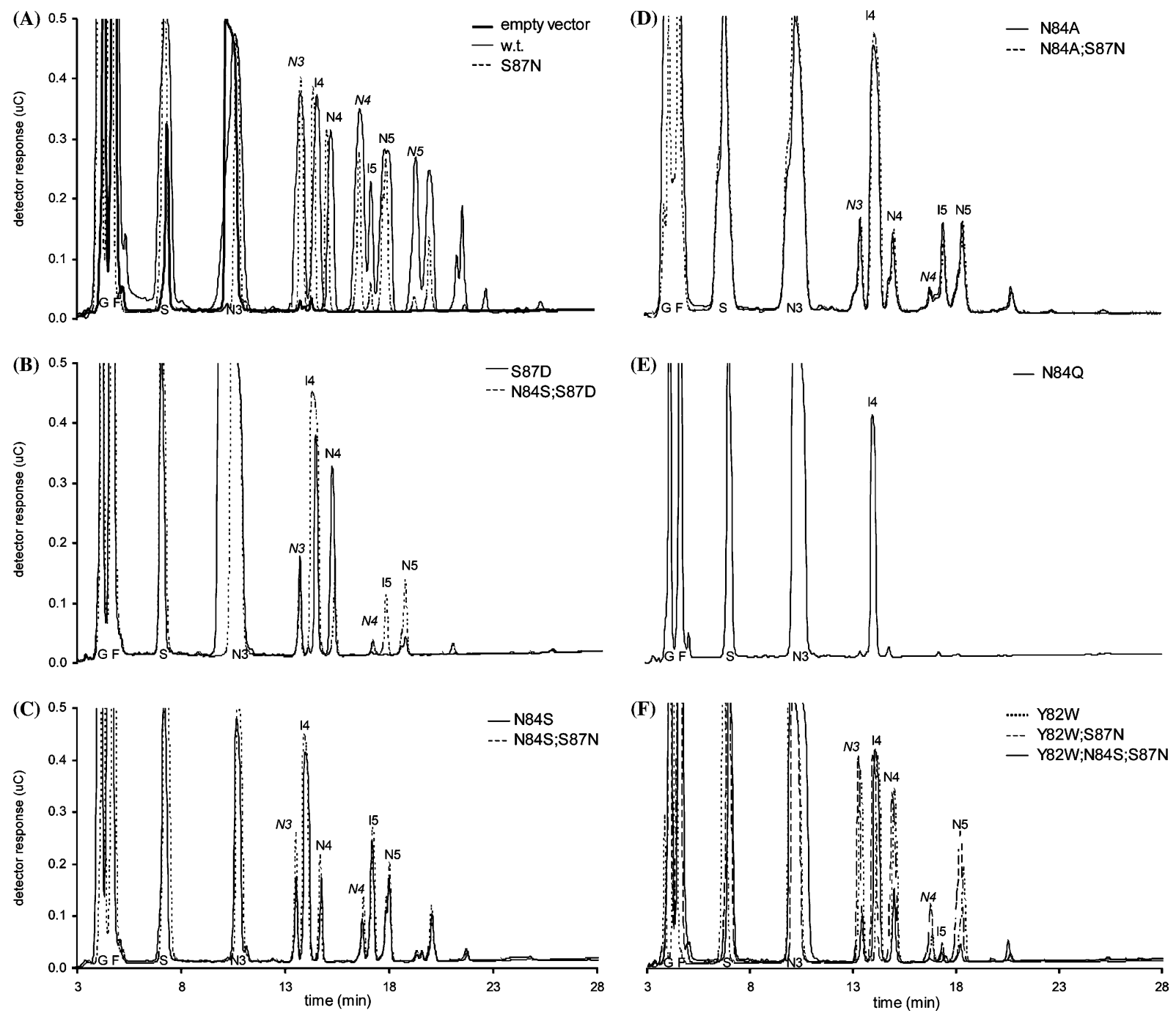

Figure 2. HPAEC-PAD profile of fructans synthesized by 6G-FFT wild type and mutants. (A) empty vector, wild type 6G-FFT and mutant S87N; (B) mutants S87D and N84S;S87D; (C) mutants N84S and N84S;S87D; (D) mutants N84A and N84A;S87N; (E) mutant N84Q; (F) mutants Y82W, Y82W;S87N, and Y82W;N84S;S87N. Extracts from BY2 cells were incubated overnight at $28{ }^{\circ} \mathrm{C}$ with $200 \mathrm{mM}$ 1-kestose. Indicated are the substrate 1-kestose (N3) and break-down products sucrose (S); fructose (F); and glucose $(\mathrm{G})$ which can also be seen in the empty vector control. Peak annotation of polymerization products according to Ernst et al. (1998) N3, 6G-kestotriose; I4, 1,1-kestotetraose; N4, 1,6G-kestotetraose; N4, 1\&6G-kestotetraose; I5, 1,1,1-kestopentaose; N5, 1,1,6G-kestopentaose; N5, mixture of 1,1\&6G-kestopentaose and 1\&1,6G-kestopentaose. Longer DP fructans are not annotated, since they are not well separated on the HPAEC-PAD system.

4 days. No polymerization of 1,1-kestotetraose was observed (data not shown), indicating that 6G-FFT N84Q is only able to polymerize 1kestose to 1,1-kestotetraose.

All mutants were also tested with sucrose as the only substrate and none of the mutants was able to synthesize products from sucrose (data not shown).

Taking these results together we conclude that Asn84 is important in determining product specificity of 6G-FFT, since mutants N84S, N84A, and
N84Q shift product preference towards synthesis of inulin-type fructans (Ix-series).

\section{Product-profiles of $6 G-F F T$ mutants}

When product formation is compared there is a clear distinction between the 6G-FFT mutants carrying the mutations N84S, N84A, or N84Q and the other mutants. Mutants carrying N84S, N84A, or N84Q have a preference for the synthesis of 


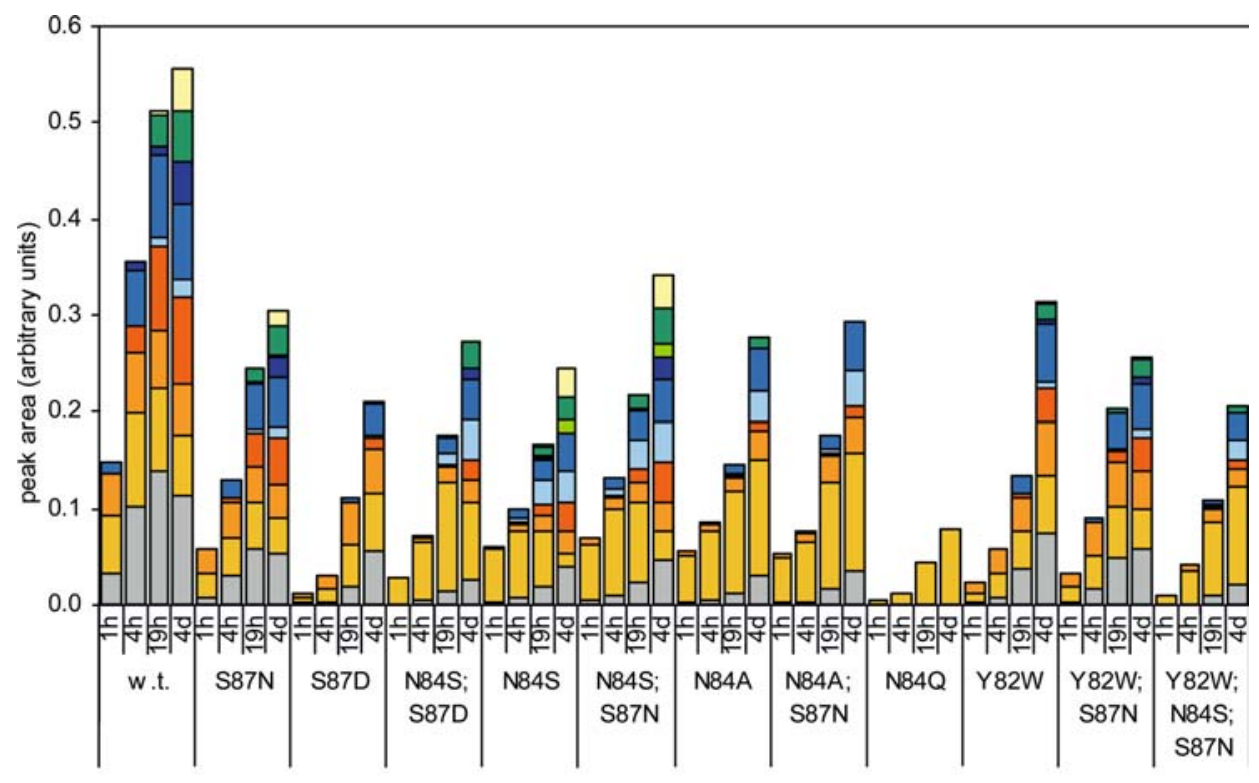

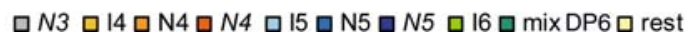

Figure 3. Time series of fructan synthesis by 6G-FFT and its mutants. Incubation times were $1 \mathrm{~h}, 4 \mathrm{~h}, 19 \mathrm{~h}$, or 4 days at $28^{\circ} \mathrm{C}$ with $200 \mathrm{mM}$ 1-kestose. Peak areas are measured in arbitrary units. Peaks: N3, 6G-kestotriose; I4, 1,1-kestotetraose; N4, 1,6G-kestotetraose; N4, 1\&6G-kestotetraose; I5, 1,1,1-kestopentaose; N5, 1,1,6G-kestopentaose; N5, mixture of 1,1\&6G-kestopentaose and 1\&1,6G-kestopentaose; I6, 1,1,1,1-kestohexose; DP6, mixture of DP6 fructans with chain elongation on both sites of the starter sucrose; rest, longer fructans.

inulin-type fructans (Ix), whereas the others synthesize the three onion fructan series Ix, Nx and $N x$ (Figure 1). The preference of the transfer of a fructose to the terminal fructose via a $\beta(2-1)$ link or to the terminal glucose via a $\beta(2-6)$ link was investigated in a more quantitative way. For this

Table 3. Overview of the activities of 6G-FFT mutants.

\begin{tabular}{|c|c|c|c|}
\hline & Active & Ix & $\mathrm{Nx} / N x$ \\
\hline w.t. & + & + & + \\
\hline Y82W & + & + & + \\
\hline Y82S & - & - & - \\
\hline N84S & + & + & - \\
\hline N84A & + & + & - \\
\hline $\mathrm{N} 84 \mathrm{G}$ & - & - & - \\
\hline N84Y & - & - & - \\
\hline N84Q & + & $+*$ & - \\
\hline D85N & - & - & - \\
\hline P86A & + & + & + \\
\hline S87N & + & + & + \\
\hline S87D & + & + & + \\
\hline Y91S & - & - & - \\
\hline Y82W;S87N & + & + & + \\
\hline N84S;S87N & + & + & - \\
\hline N84S;S87D & + & + & - \\
\hline N84A;S87N & + & + & - \\
\hline N84Y;S87N & - & - & - \\
\hline Y82W;N84S;S87N & + & + & - \\
\hline
\end{tabular}

Indicated is overall activity and the type of fructans made; Ix, inulin-type fructans; Nx/Nx, neo-series fructans (see Figure 1). *This mutant makes only I4 (GFFF). 
the amounts of 1,1-kestotetraose (I4) over 1\&6Gkestotetraose (N4) were determined by measurement of the peak areas after $1 \mathrm{~h}$ of incubation with 1-kestose. This short incubation-time resulted in no other detectable polymerization products than 1,1-kestotetraose and 1\&6G-kestotetraose. Mutants with a preference for inulin-type fructans showed less than $10 \% 1 \& 6 \mathrm{G}$-kestotetraose formation after $1 \mathrm{~h}$ and more than 90\% 1,1-kestotetraose (Figure 4). In contrast, mutants that had a product profile comparable to the wild-type enzyme showed approximately 50\% 1\&6G-kestotetraose and 50\% 1,1-kestotetraose formation (Figure 4). No intermediate distributions were found, indicating that the transfer of fructose to the glucose residue is a distinct phenomenon.

The presumed nucleophile Asp85 the $\beta$-fructosidase motif of $6 G-F F T$ is essential for activity

Mutations of the proposed nucleophile of yeast invertase and bacterial fructosyltransferases resulted in inactive enzymes (Reddy and Maley, 1996; Ozimek et al., 2004). The proposed nucleophile of plant fructosyltransferases is the Asp present in the $\beta$-fructosidase motif of the sucrosebinding box. It is invariable in vacuolar invertases and plant fructosyltransferases indicating that also in 6G-FFT the corresponding amino acid is essential for activity. Indeed, changing Asp85 to Asn resulted in an inactive enzyme (Table 3).

The directly neighboring Pro 86 is also an invariable amino acid (Table 1 and 2). Mutating Pro86 into Ala resulted in a 6G-FFT with wild-type specificity, although transfructosylation was slow. Overnight incubation with 1-kestose showed two small peaks, 1,1-kestotetraose (I4) and 1,6G-kestotetraose (N4) (data not shown). Thus activity seems severely reduced, but a wild-type product-profile is observed (Table 3 ).

\section{Mutations in aromatic residues surrounding the $\beta$-fructosidase motif}

To investigate the function of the aromatic amino acids surrounding the $\beta$-fructosidase motif in the binding of sugar substrates, five additional mutants were made. In the 6G-FFT mutant Y82W Trp is present at the second residue $\mathrm{N}$-terminal of the $\beta$-fructosidase motif, this is according to the sucrose-binding box of vacuolar invertases (Table 1). Mutant $\mathrm{Y} 82 \mathrm{~W}$ shows no change in substrate or product preference (Figures 2F, 3, and 4). A similar result was observed when the double mutant $\mathrm{Y} 82 \mathrm{~W} ; \mathrm{S} 87 \mathrm{~N}$ was tested (Figures $2 \mathrm{~F}, 3$, and 4, Table 3). However, the triple mutant Y82W;N84S;S87N showed a changed product preference towards Ix-type fructans. Thus it has the same product profile as the N84S mutant (Table 3). These results indicate that the $\beta$-fructosidase motif in itself determines product preference of $6 \mathrm{G}-\mathrm{FFT}$. The nature of the aromatic amino acid preceding the $\beta$-fructosidase motif seems not involved in this.

The 6G-FFT mutant Y82S, in which the polar aromatic amino acid is replaced for a polar non-aromatic residue, is inactive (Table 3 ). Tyr91, which is the first aromatic amino acid

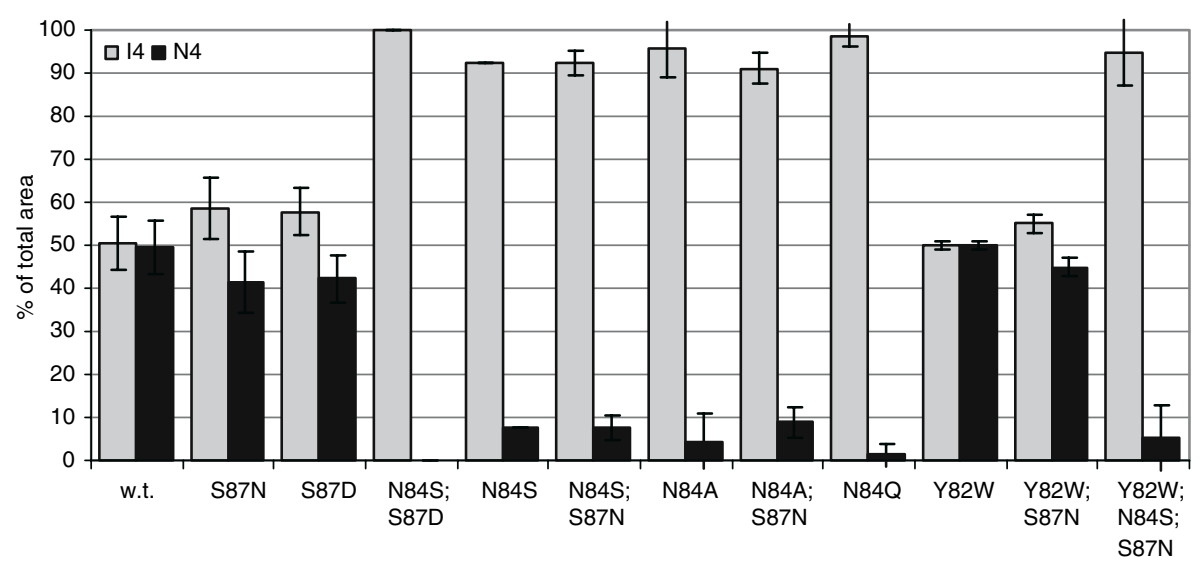

Figure 4. Initial fructosyl transfer by 6G-FFT and its mutants using 1-kestose as a substrate represented as a $\%$ of the total area of polymerization products \pm S.D. $\mathrm{N}=2,3$ or 4 . I4, 1,1-kestotetraose; $N 4,1,6 \mathrm{G}$-kestotetraose. 
following the $\beta$-fructosidase motif of $6 \mathrm{G}-\mathrm{FFT}$, was also mutated into Ser. This led to an inactive enzyme as well (Table 3). These results indicate that the aromatic residues surrounding the $\beta$-fructosidase motif are essential for fructosyltransferase activity.

\section{Discussion}

Natural variation observed in the sucrose-binding box of fructosyltransferases is predictive for their functionality. For example, dicot 1-SSTs contain the $\beta$-fructosidase motif SDPDG, 6-SFTs have SDPNG, and 1-FFTs have YDPN/DG (Table 1). We designed our mutants according to the natural alterations observed, with an emphasis on the nucleophile-containing $\beta$-fructosidase motif. The aim was to test the role of individual amino acids in determining the specificity of the fructosyltransferase 6G-FFT via mutational analysis using the variation found in nature as a guide. Changing the $\beta$-fructosidase motif of onion 6G-FFT to that of 1FFT eliminated the function of the enzyme, whereas the $\beta$-fructosidase motif of 6-SFT resulted in inulin (Ix) synthesis, i.e. 1-FFT activity (Figure 1, Tables 1-3). Similarly, dicotyledonous or onion 1-SST motifs led to 1-FFT activity. The vacuolar invertase and grass 1-SST $\beta$-fructosidase motif yielded 6G-FFT activity, since the full spectrum of 6G-FFT-dependent fructans (Ix, Nx, and $N x$ ) (Figure 1, Tables 1-3) was produced. 1-SSTs and 6-SFTs can use sucrose as their sole substrate, but none of the 6G-FFT mutants could use sucrose for fructosyl transfer.

Recently another paper was published on enzymatic specificity of fructosyltransferases. This paper compares a 6-SFT and 1-SST, fructosyltransferases that can use both sucrose and 1kestose, and it shows that the N-terminal 2/3 of the protein, which includes the sucrose-binding box, determines catalytic specificity (Altenbach et al., 2004). This observation is in agreement with our results, since we show that even a single amino acid in the sucrose-binding box can influence the products synthesized.

Bacterial fructosyltransferases are supposed to work via a ping-pong mechanism in which the fructosyldonor is bound, the fructose is split off and stays bound while the rest of the donor molecule is released, then the fructosylacceptor is bound, the fructose linked and the newly synthesized molecule released (Chambert et al., 1974). Likely, this is also the catalysis mechanism of fructosyltransferases. Only one sucrose-binding box is identified in fructosyltransferases, which is probably used by both donor and acceptor substrates. Furthermore, we show here that the sucrose-binding box is involved in product specificity, indicating that the acceptor substrate is binding at the sucrose-binding box (which was identified as the donor substrate binding site in invertases).

Invertases are retaining glycosidases (Alberto et al., 2004), meaning that their anomeric configuration is retained during hydrolysis. In these enzymes the fructose residue remains in the active site after hydrolysis of sucrose, while the glucose residue is released. Subsequently, a reaction with water releases the fructose residue. This reaction mechanism would already form the basis for the ping-pong mechanism to occur in fructosyltransferases during fructose polymerization.

Both Asp residues of the $\beta$-fructosidase motif are highly variable in fructosyltransferases. Changing Ser87 in Asn(N) or Asp(D) did not change product preference of the enzyme. Changing $\mathrm{Asn} 84$ in $\operatorname{Ser}(\mathrm{S})$, $\mathrm{Ala}(\mathrm{A})$, or $\mathrm{G} \ln (\mathrm{Q})$ did, however, shift product specificity towards Ix series fructans. N84S combined with $\mathrm{S} 87 \mathrm{~N}$, or S87D showed similar product specificities as N84S alone did. Also combining N84A with S87N did result in the same profile as N84A. So the identity of the amino acid at position 84 determines the substrate to which the fructose is transferred. In accordance with this, mutants S84Y and S84G were not polymerizing at all. In conclusion, N84 seems essential for the array of products made by $6 \mathrm{G}-$ FFT; i.e. Ix, $N x$, and $\mathrm{Nx}$-series fructans. The other amino acids tested on position 84 narrow the products made to either Ix series fructans or none at all.

The only fructosyltransferase from which we know the 3 dimensional structure is levansucrase from the bacterium Bacillus subtilis (Meng and Futterer, 2003). This enzyme belongs to glycosyl hydrolase family 68 , although it is mechanistically similar to plant fructosyltransferases. The recently published GH32 structures of invertase from the bacterium Thermotoga maritima (Alberto et al., 2004), exo-inulinase from the fungus Aspergillus awamori (Nagem et al., 2004), and fructan 
exohydrolase from the plant Cichorium intybus (Verhaest et al., 2005) allow modeling of the sucrose binding box of onion 6G-FFT. These hydrolyzing enzymes have the $\beta$-fructosidase motif NDPNG. In the $\beta$-fructosidase box of 6G-FFT, we see that the second Asn is replaced by Ser. In $T$. maritima invertase the sucrose was modeled in the active site, in A. awamori exo-inulinase the fructose was crystallized in the active site, and in FEH a glycerol was bound instead of a sugar. In all these examples the Asn preceding the nucleophilic Asp was in contact with the bound molecule, but the second Asn is not. Extrapolating these observations one can assume that the 6G-FFT Serresidue of the $\beta$-fructosidase motif is also not in contact with the substrate 1-kestose. This would explain why changing this residue has little effect on the product preference of 6G-FFT. Even though the Ser remains an intriguing amino acid substitution in such a very conserved region.

In the $\beta$-fructosidase motif of $6 \mathrm{G}$-FFT the presumed nucleophile Asp85 is present. This residue is conserved among $\beta$-fructosidases/fructosyltransferases. In invertases and bacterial fructosyltransferases this amino acid was proven to be essential for activity (Reddy and Maley, 1996; Ozimek et al., 2004). Removing the negative charge by changing Asp85 to Asn rendered 6GFFT non-active. This indicates the essential nature of this conserved negatively charged amino acid for enzyme activity in plant fructosyltransferases.

From in silico analysis, other amino acids of the sucrose-binding box could be related to enzyme identity. In invertases Trp is present as the second amino acid $\mathrm{N}$-terminal of the $\beta$-fructosidase motif. In fructosyltransferases no $\operatorname{Trp}$ is found at this position, but the aromatic amino acids Tyr or Phe are present. Aromatic amino acids are often involved in binding of sugars, and it was shown that the Trp directly preceding the presumed nucleophile Asp is involved in binding of the substrate sucrose in bacterial fructosyltransferases (Meng and Futterer, 2003). There is also a Trp in the GH32 enzymes crystallized at two amino acids $\mathrm{N}$-terminal of the active-site Asp, but it seems that this Trp is not involved in substrate binding (Alberto et al., 2004; Nagem et al., 2004; Verhaest et al., 2005).

In onion $6 \mathrm{G}-\mathrm{FFT}$ the aromatic residue Tyr82 is two amino acids $\mathrm{N}$-terminal of the presumed nucleophilic Asp. Substituting Tyr82 for Trp did not alter enzyme specificity. Also when in the mutant S87N, Y82W was introduced, activity remained unchanged. Introduction of $\mathrm{Y} 82 \mathrm{~W}$ in the double-mutant N84S;S87N, which synthesizes Ix-type fructans, did also not alter enzyme specificity. These mutants indicate that although a Trp residue is not present at this position in fructosyltransferases it does not affect transferase specificity of wild-type or mutated 6G-FFTs.

However, when Tyr82 was changed into a non-aromatic Ser residue activity was lost, suggesting that this amino acid is important for either enzymatic activity or protein structure. Since aromatic amino acids are often involved in sugar binding, this might also be the function of Tyr82. Its position at the rim of the active site pocket would make it ideally positioned to interact with longer substrates like fructans. Also 6G-FFT mutant Y91S in which the aromatic amino acid following the sucrose-binding box was mutated was inactive. Since this amino acid seems not to be in proximity of the active site, structural requirements are more likely the reason for its necessity.

Production of inulin neo-series proceeds if both the glucose and fructose terminal residues of the acceptor molecule are available for fructosyl transfer. Probably, the acceptor substrate can enter the enzymatic pocket in two ways, either with a terminal fructose residue or with the terminal glucose residue first. Mutations N84S, N84A and N84Q might hinder the acceptor substrate from entering the enzymatic pocket with the terminal glucose residue leading, alternatively, the substrate can enter with the glucose first, but these mutations prevent linkage of the fructose to the terminal glucose.

Enzymes showing wild-type activity synthesize approximately 50\% 1\&6G-kestotetraose and 50\% 1,1-kestotetraose within 1 hour of incubation with 1-kestose. If the activity was shifted towards Ix type fructans, less than $10 \% 1 \& 6 \mathrm{G}-$ kestotetraose and more than $90 \%$ 1,1-kestotetraose was produced (Figure 4). No intermediate distributions were observed. These distribution profiles are probably a result of the accessibility of the terminal sugar residues of the acceptor 1-kestose. A wild-type distribution of 50/50 fits with an equal availability of the terminal glucose and fructose residues. In the case of Ix preference the terminal fructose is still elongated, but transfer to the terminal glucose is blocked. 


\section{Acknowledgements}

We like to thank Dr. Masao Hirayama, Meiji Seika Kaisha Ltd., Japan for his kind gift of 1-kestose. The Dutch Organization for Scientific Research (NWO) funded this research.

\section{References}

Alberto, F., Bignon, C., Sulzenbacher, G., Henrissat, B. and Czjzek, M. 2004. The three-dimensional structure of invertase ( $\beta$-fructosidase) from Thermotoga maritima reveals a bimodular arrangement and an evolutionary relationship between retaining and inverting glycosidases. J. Biol. Chem. 279: 18903-18910.

Altenbach, D., Nuesch, E., Meyer, A.D., Boller, T. and Wiemken, A. 2004. The large subunit determines catalytic specificity of barley sucrose:fructan 6-fructosyltransferase and fescue sucrose:sucrose 1-fructosyltransferase. FEBS Lett. 567: 214-218.

An, G. 1985. High efficiency transformation of cultured tobacco cells. Plant Physiol 79: 568-570.

Chambert, R., Treboul, G. and Dedonder, R. 1974. Kinetic studies of levansucrase of Bacillus subtilis. Eur. J. Biochem. 41: 285-300.

Edelman, J. and Jefford, T.G. 1968. The metabolism of fructose polymers in plants. New Phytol. 67: 517-531.

Ernst, M., Chatterton, N.J., Harrison, P.A. and Matitschka, G. 1998. Characterization of fructan oligomers from species of the genus Allium L. J. Plant Physiol. 153: 53-60.

Fujishima, M., Sakai, H., Ueno, K., Takahashi, N., Onodera, S., Benkeblia, N. and Shiomi, N. 2005. Purification and characterization of a fructosyltransferase from onion bulbs and its key role in the synthesis of fructo-oligosaccharides in vivo. New Phytol. 165: 513-524.

Genschik, P., Criqui, M.C., Parmentier, Y., Derevier, A. and Fleck, J. 1998. Cell cycle-dependent proteolysis in plants: identification of the destruction box pathway and metaphase arrest produced by the proteasome inhibitor MG132. Plant Cell 10: 2063-2075.

Hendry, G.A.F. 1993. Evolutionary origins and natural functions of fructans - a climatological, biogeographic and mechanistic appraisal. New Phytol. 123: 3-14.

Koops, A.J. and Jonker, H.H. 1996. Purification and characterization of the enzymes of fructan biosynthesis in tubers of Helianthus tuberosus Colombia. II. Purification of sucrose:sucrose 1-fructosyltransferase and reconstitution of fructan synthesis in vitro with purified sucrose:sucrose 1-fructosyltransferase and fructan:fructan 1-fructosyltransferase. Plant Physiol. 110: 1167-1175.

Lüscher, M., Erdin, C., Sprenger, N., Hochstrasser, U., Boller, U. and Wiemken, A. 1996. Inulin synthesis by a combination of purified fructosyltransferase from tubers of Helianthus tuberosus. FEBS lett. 385: 39-42.

Meng, G. and Futterer, K. 2003. Structural framework of fructosyl transfer in Bacillus subtilis levansucrase. Nat. Struct. Biol. 10: 935-941.

Nagata, T., Nemoto, Y. and Hasazawa, S. 1992. Tobacco BY2 cell line as the "HeLa" cell in the cell biology of higher plants. Int. Rev. Cytol. 132: 1-30.
Nagem, R.A., Rojas, A.L., Golubev, A.M., Korneeva, O.S., Eneyskaya, E.V., Kulminskaya, A.A., Neustroev, K.N. and Polikarpov, I. 2004. Crystal structure of exo-inulinase from Aspergillus awamori: the enzyme fold and structural determinants of substrate recognition. J. Mol. Biol. 344: 471-480.

Ozimek, L.K., Hijum, S.A.Van, Koningsveld, G.A.van, Maarel, M.J.Der, Geel-Schutten, G.H. and Dijkhuizen, L. 2004. Site-directed mutagenesis study of the three catalytic residues of the fructosyltransferases of Lactobacillus reuteri 121. FEBS Lett. 560: 131-133.

Pons, T., Hernandez, L., Batista, F.R. and Chinea, G. 2000. Prediction of a common beta-propeller catalytic domain for fructosyltransferases of different origin and substrate specificity. Protein Sci. 9: 2285-2291.

Reddy, A. and Maley, F. 1990. Identification of an active-site residue in yeast invertase by affinity labeling and sitedirected mutagenesis. J. Biol. Chem. 265: 10817-10820.

Reddy, A. and Maley, F. 1996. Studies on identifying the catalytic role of Glu-204 in the active site of yeast invertase. J. Biol. Chem. 271: 13953-13957.

Ritsema, T., Joling, J. and Smeekens, S. 2003. Fructan patterns synthesized by onion fructan:fructan $6 \mathrm{G}$-fructosyltransferase (6G-FFT) expressed in tobacco BY2 cells - is fructan:fructan 1-fructosyltransferase (1-FFT) needed in onion?. New Phytologist 160: 61-67.

Ritsema, T., Verhaar, A., Vijn, I. and Smeekens, S. 2004. Fructosyltransferase mutants specify a function for the $\beta$-fructosidase motif of the sucrose-binding box in specifying the fructan type synthesized. Plant Mol. Biol. 54: 853-863.

Roberfroid, M. 2002. Functional food concept and its application to prebiotics. Dign. Liver Dis. 34(Suppl 2) S105-S1 10.

Shiomi, N. 1993. Structure of fructopolysaccharide (asparago$\mathrm{sin}$ ) from roots of asparagus (Asparagus officinalis L.). New Phytol. 123: 263-270.

Shiomi, N., Onodera, S. and Sakai, H. 1997. Fructo-oligosaccharide content and fructosyltransferase activity during growth of onion bulbs. New Phytol. 136: 105-113.

Sprenger, N., Bortlik, K., Brandt, A., Boller, T. and Wiemken, A. 1995. Purification, cloning, and functional expression of sucrose:fructan 6-fructosyltransferase, a key enzyme of fruc$\tan$ synthesis in barley. Proc. Natl. Acad. Sci. USA 92: 1165211656.

Van den Ende, W. and Van Laere, A. 1996. De-novo synthesis of fructans from sucrose in vitro by a combination of two purified enzymes (sucrose:sucrose 1-fructosyltransferase and fructan:fructan 1-fructosyltransferase) from chicory roots (Cichorium intybus L.). Planta 200: 335-342.

Verhaest, M., Van den Ende, W., Roy, K.L., De Ranter, C.J., Laere, A.V. and Rabijns, A. 2005. X-ray diffraction structure of a plant glycosyl hydrolase family 32 protein: fructan 1-exohydrolase IIa of Cichorium intybus. Plant J. 41: 400-411.

Vijn, I., van Dijken, A., Sprenger, N., van Dun, K., Weisbeek, P., Wiemken, A. and Smeekens, S. 1997. Fructan of the inulin neoseries is synthesized in transgenic chicory plants (Cichorium intybus L.) harbouring onion (Allium cepa L.) fructan:fructan 6G-fructosyltransferase. Plant J. 11: 387-398.

Waterhouse, A.L. and Chatterton, N.J. 1993. Glossary of fructan terms. In: M. Suzuki and N.J. Chatterton (Eds.), Science and Technology of Fructans, CRC Press, Boca Raton, FL, pp. 1-8.

Wei, J.Z. and Chatterton, N.J. 2001. Fructan biosynthesis and fructosyltransferase evolution: expression of the 6-SFT (sucrose:fructan 6-fructosyltransferase) gene in crested wheatgrass (Agropyron cristatum). J. Plant Physiol. 158: $1203-1213$. 